\title{
Review
}

\section{Rare Infections of the Oral Mucosa}

\author{
Ioanina Părlătescu *, Radu Cazacu and Șerban Țovaru \\ Oral Pathology Discipline, Faculty of Dental Medicine, Carol Davila University of Medicine and Pharmacy, \\ 4-6 Eforie St, 050037 Bucharest, Romania; cazacu.radu@outlook.com (R.C.); serban.tovaru@gmail.com (S.T.) \\ *Correspondence: ioanina.parlatescu@umfcd.ro; Tel.: +40-724950104
}

Submitted: 2 December 2020; Accepted: II January 202I; Published: 20 January 202I

\begin{abstract}
I) Background: Oral mucosal infections are a commonly encountered clinical pathology in patients who consult dentists, mainly for dental diseases. Currently, the involvement of oral and dental infections in cardiovascular and neurological diseases, among others, is widely discussed in the literature. In the present research, we elaborated a comprehensive report on rare oral mucosal infections, including oral syphilis and head and neck tuberculosis. (2) Methods: The research was performed by the authors using three databases (PubMed, Wiley, and ScienceDirect). Scientific articles published from January 2000 to January 2020 were selected and reviewed to assess the epidemiology, symptoms, and diagnosis of oral syphilis and head and neck tuberculosis. (3) Results: Oral syphilis was mainly noticed as ulcers and secondary types of disease. Tuberculosis of the head and neck was most frequently encountered as cervical lymphadenopathy. (4) Conclusions: Rare oral infections diagnosis may be challenging due to their evolving polymorphic clinical appearance and their ability to mimic various diseases in the oral cavity and head and neck area.
\end{abstract}

Keywords: oral infections; syphilis; tuberculosis

How to cite: Părlătescu, I.; Cazacu, R.; T,ovaru, Ș. Rare Infections of the Oral Mucosa. Timisoara Med. 2020, 2020(2), 2; doi:10.35995/tmj20200202.

\section{Introduction}

Oral mucosal infections are a commonly encountered clinical pathology in patients who consult dentists, mainly for dental diseases. The predisposing factors for oral mucosal infections are general as well as local. Among the general factors, associated diseases such as diabetes, cardiovascular disorders, and chronic medication have special importance. Local factors that influence oral mucosal infections are smoking habits, reduced oral hygiene, the presence of mucosal lesions that facilitate pathogens access in the body, and the presence of dental and periodontal foci [I]. Currently, the involvement of oral and dental infections in cardiovascular and neurological diseases, among others, is widely discussed in the literature. The pathogens causing oral infections are fungi, bacteria, and viruses. In the present research, we elaborated a comprehensive report on rare oral mucosal infections, including oral syphilis and head and neck tuberculosis.

Syphilis is a chronic worldwide sexually transmitted infection caused by Treponema pallidum [2]. Humans are the only proven host. The microorganism is anaerobic, does not have resistance in a dry environment, and cannot survive outside the body, which is why its spread is almost exclusively sexual or can be transmitted 
from mother to fetus. Syphilis was frequently encountered in the early zoth century and was determined to cause heart and neurological system disorders [3]. After the discovery of penicillin therapy in the r940s, the prevalence of syphilis slowly decreased for several years. A peak occurred during the "sexual revolution" of the r96os, but fears of acquired immunodeficiency (AIDS) in 2000 led to the fewest cases of primary and secondary syphilis reported since 1941. As more effective AIDS therapy has been developed, sexual activity has increased, with an increasing prevalence of sexually transmitted diseases, especially among homosexuals [2]. Syphilis diagnoses reported worldwide in 2008 were 36.4 million infected adults and Io.6 million new cases $[4,5]$. Although there has been a progressive reduction in syphilis prevalence $[3,6]$ in the last two decades, the disease has reappeared in North America, Europe, Russia, and China. Indiscriminate sexual behavior, declining use of sexual safety methods, and HIV coinfection are the factors blamed for changes in the epidemiology of syphilis.

Tuberculosis is an infectious disease produced by Mycobacterium tuberculosis. This chronic condition remains a considerable public health problem even in countries which have a well-developed healthcare system. Tuberculosis commonly affects the lungs, but can also involve any organ. One in five cases of tuberculosis have an extrapulmonary form (the intestines, meninges, bones, joints, lymph glands, skin, and other tissues of the body) $[7,8]$. Although extrapulmonary occurrence is genetically determined, immunosuppression of various causes can also favor the development of extrapulmonary involvement, from $16 \%$ in 1993 in the US to $21 \%$ of all tuberculosis cases in 2005 [8].

The aim of this work was to summarize the research evidence of the epidemiological and clinical characteristics of oral syphilis and head and neck tuberculosis.

\section{Materials and Methods}

For the present study, two systematic research studies were performed to analyze data from the literature on published studies for oral tuberculosis and syphilis. The research was performed by the authors using three databases (PubMed, Wiley, and ScienceDirect). Scientific articles published from January 2oor to December 2019 were selected and reviewed to assess the epidemiology, symptoms, and diagnosis of oral syphilis and head and neck tuberculosis. The flowchart of the methodology is presented in Figure $\mathrm{I}$. In the first phase, the articles were evaluated based on the title and abstract. In the second phase, the content of the articles was evaluated to determine whether the studies included a number of 20 patients for syphilis and 40 patients for head and neck tuberculosis. This was conducted by two authors. The full texts of the potentially relevant studies were retrieved and evaluated. The reference lists of the selected articles were searched manually to identify any other relevant studies that were overlooked using the search strategy.

For the search related to syphilis, the keywords used were "syphilis" and "oral syphilis". The initial search led to Io,I6o published studies. The studies were eligible for this paper if they presented oral manifestations of syphilis, as well as if the lesions' site was mentioned and a number of at least 20 participants were included in the study. For the present research, we retained data from three studies which comprised more than 20 participants [9-II].

For the search related to tuberculosis in the databases, the keywords were "tuberculosis" and "head and neck". The initial search led to 626I published studies. We excluded irrelevant studies concerning treatment, case reports, and editorials. For the present research, we retained data from three studies with more than 40 participants [I2-I4]. 
Demographic data, age, gender, time of onset, symptoms, lesion site, type and size of the lesion, as well as the stage of syphilis (primary, secondary, or tertiary) were extracted and tabulated.

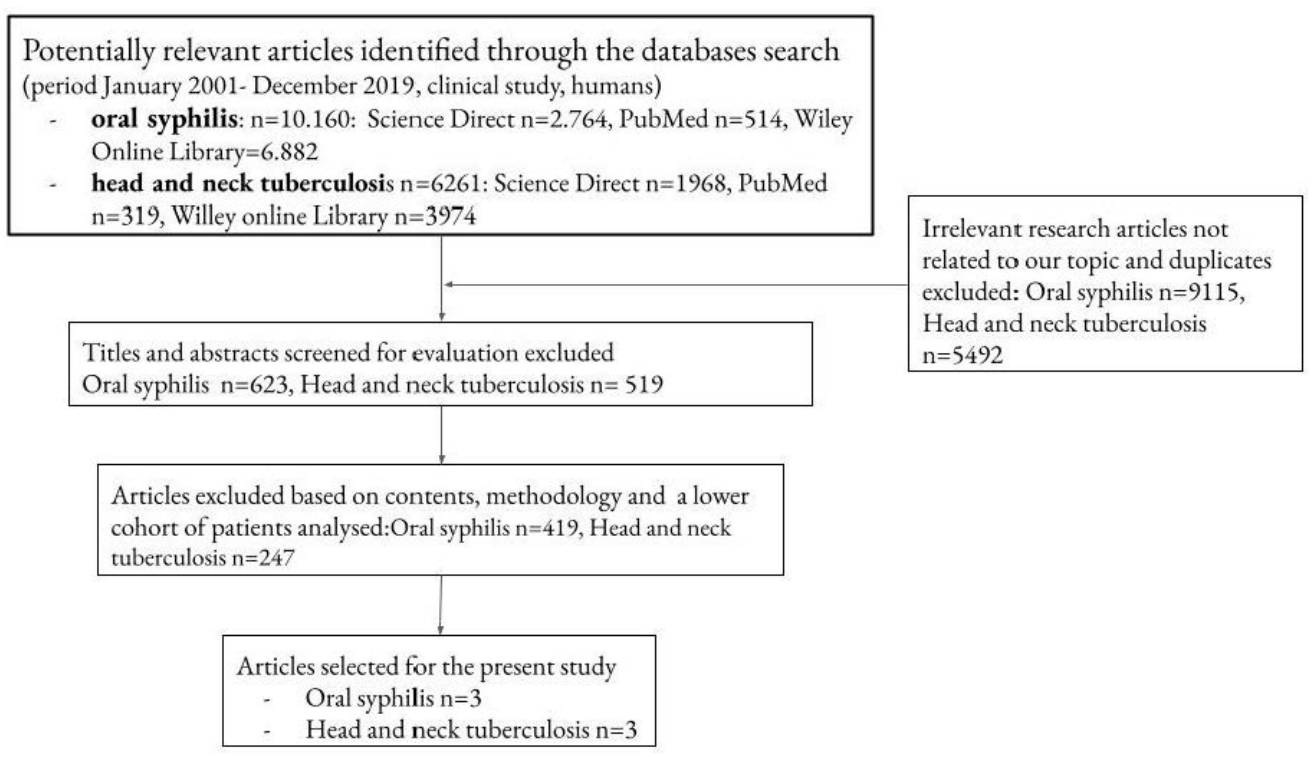

Figure 1. Flowchart of the review selection methodology.

\section{Results}

\section{Oral Syphilis}

A total of I59 patients were included in the selected studies, of which there were Ioo men representing $62.89 \%$ and a total of 59 women representing 37.II\% (Figure 2). Single oral lesions were noticed in I29 patients $(82.2 \%)$ and were represented by ulcerations, while multiple lesions were identified in 28 patients (I7.8\%) (Table I). From the analyzed data, pain was reported by II patients, 2 patients reported burning sensations, while 7 cases were identified as asymptomatic. The location of the lesions was divided into the following categories: lips, labial commissure, palate, tongue, buccal mucosa, jugal mucosa, pharynx, gum, and alveolar ridge. The tongue was the most common location ( 53 cases), followed closely by the lips ( $5 \mathrm{I}$ cases), palate (20 cases), buccal mucosa (I4 cases), floor of the mouth (I3 cases), labial commissure (Io cases), pharynx and palatine amygdala ( 6 cases each), alveolar ridge ( 4 cases), gingiva ( 1 case), and 5 patients where the lesion site was not specified (Figure 3 ). The majority of patients (85.5\%) had secondary syphilis $(n=136), 12.6 \%$ had primary syphilis $(n=20)$ and $\mathrm{I} .9 \%$ had tertiary syphilis $(n=3)$. The diagnosis of syphilis was made based on clinical data and serological tests. Following additional serological tests, 29 patients were identified as HIV-positive (Table I).

Table 1. Data of analyzed studies on oral syphilis.

\begin{tabular}{llll}
\hline & Study $\mathbf{1}$ & Study 2 & Study $\mathbf{3}$ \\
\hline Year of the publication and citation & $2019,[9]$ & $2013,[\mathrm{IO}]$ & $2020,[\mathrm{II}]$ \\
Total number of patients & 40 & 34 & 85 \\
HIV association & $\mathrm{I}$ & $\mathrm{O}$ & 28 \\
Primary syphilis & 3 & $\mathrm{I} 2$ & 5 \\
Secondary syphilis & 37 & $\mathrm{I} 9$ & $8 \mathrm{O}$ \\
Tertiary syphilis & $\mathrm{O}$ & 3 & $\mathrm{O}$ \\
Single/multiple oral lesions & $\mathrm{I} 8 / 20$ & $29 / 5$ & $82 / 3$ \\
\hline
\end{tabular}




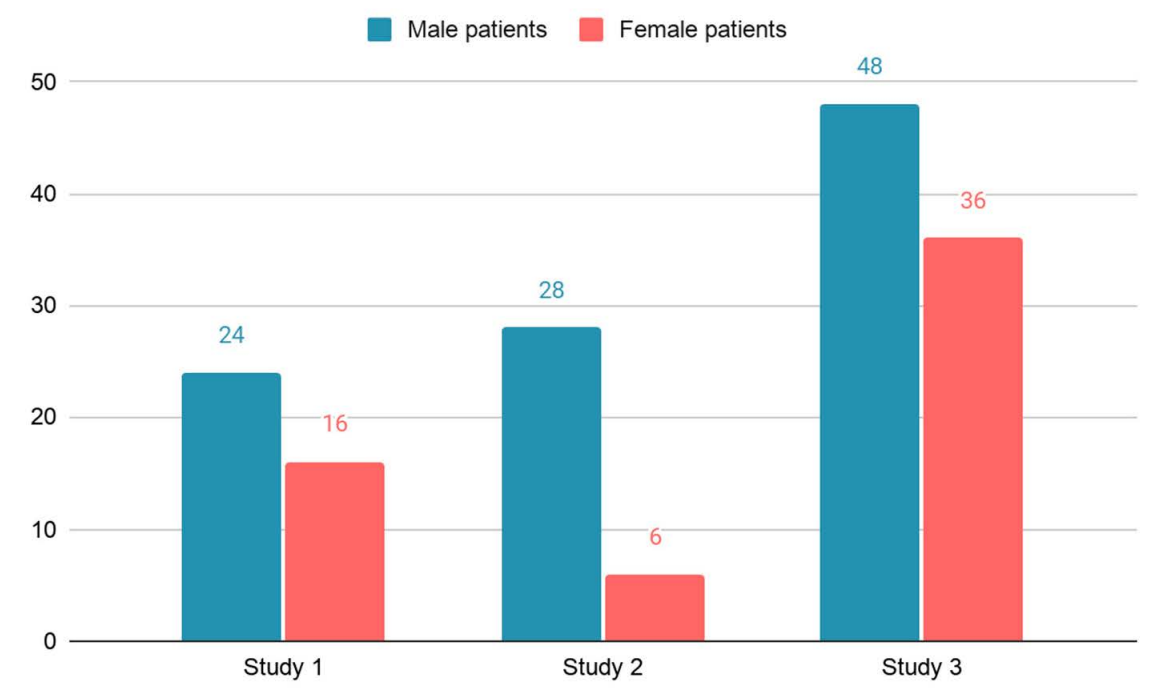

Figure 2. Gender distribution of the patients diagnosed with oral syphilis.

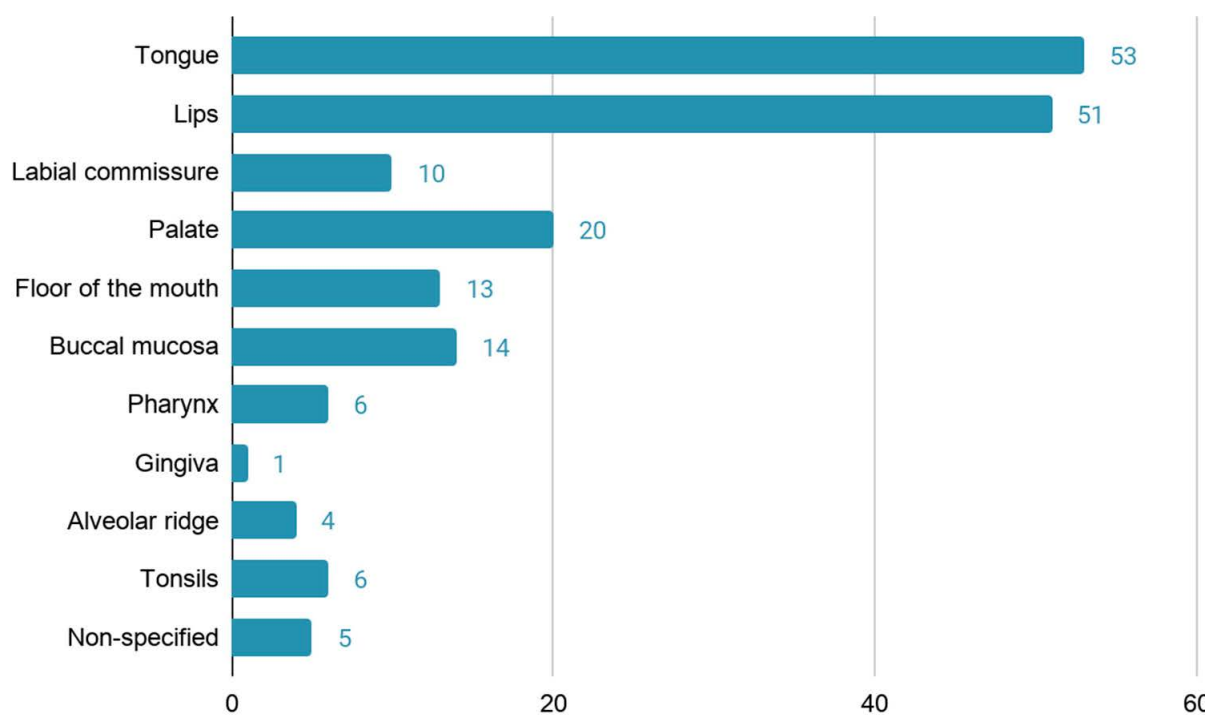

Figure 3. Location of oral syphilis lesions.

\section{Tuberculosis}

The total number of patients included in the three selected studies is 680 cases. Of these, 299 were men, representing $43.97 \%$, and 381 were women $(56.03 \%$ ) (Figure 4 ). A percentage of I.6I\% of patients had a history of tuberculosis in the family or contact with tuberculosis patients, and $3.67 \%$ were previously diagnosed with tuberculosis (Table 2). Interestingly, $22 \%$ of patients with excessive alcohol and drug use and all HIV-positive patients had a higher frequency of association of head and neck and pulmonary tuberculosis. Cervical lymphadenopathy was present in $92 \%$ of cases, followed by a frequency of laryngeal tuberculosis at $4 \%$, in the salivary gland at $3 \%$, pharynx at $0.73 \%$, and one case located in the submandibular gland, tongue, nose, parotid gland, and ear (Figure 5). Of the total reports of head and neck tuberculosis included in the present analysis, $43.5 \%$ were from North America (28\% United States, $15.5 \%$ Mexico), 10\% from Europe, $9.5 \%$ India, $7 \%$ Vietnam, $3 \%$ Philippines, and the remaining $27 \%$ from other unspecified locations. 


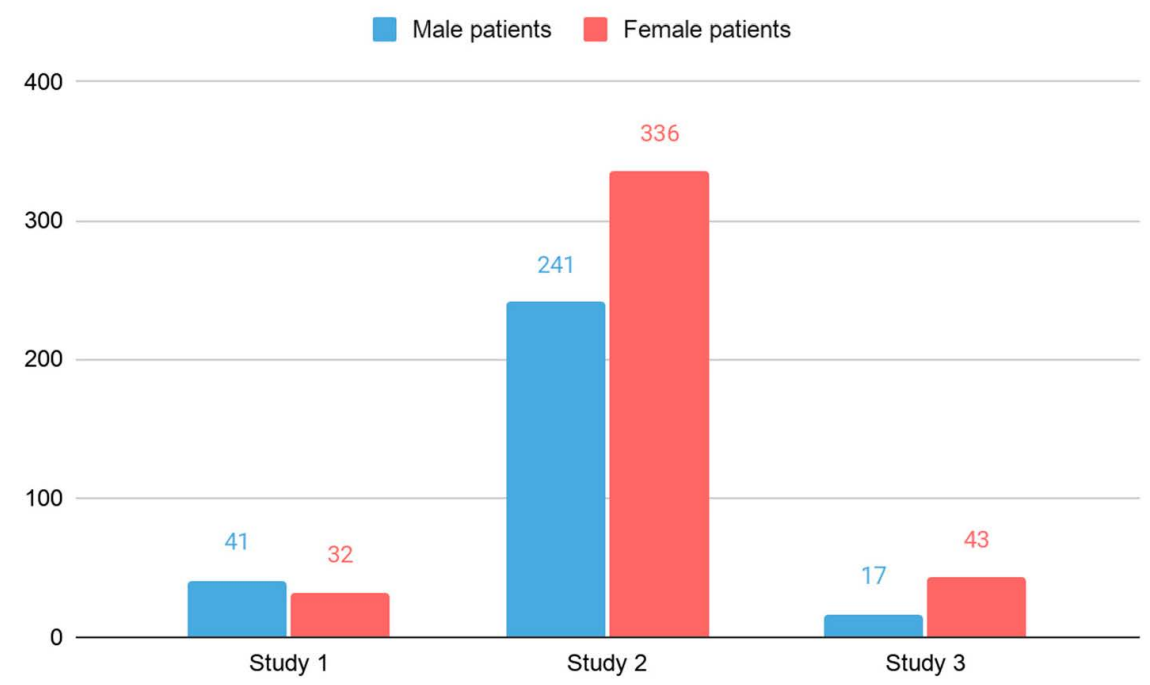

Figure 4. Gender distribution of the patients diagnosed with tuberculosis.

Table 2. Data of analyzed studies on tuberculosis.

\begin{tabular}{llll}
\hline & Study 1 & Study 2 & Study 3 \\
\hline Year of the publication and citation & $2016,[\mathrm{I} 2]$ & $2019,[\mathrm{I} 3]$ & 2018, [I4] \\
Total number of patients & 73 & 547 & 60 \\
Percentage male/female & $56.2 / 43.8$ & $56 / 44$ & $35.3 / 74.7$ \\
Average age & 39.4 & not specified & 45 \\
& Mantoux test, & Pulmonary & Pulmonary radiography, \\
Diagnostic tests & Lowenstein-Jensen culture, & radiography & Mantoux test, \\
& Ziehl-Neelsen staining, PCR & & histopathology \\
HIV association & I4 & 64 & o \\
Previous tuberculosis contact & IO & O & I \\
Previous tuberculosis disease & 2 & I2 & II \\
\hline
\end{tabular}

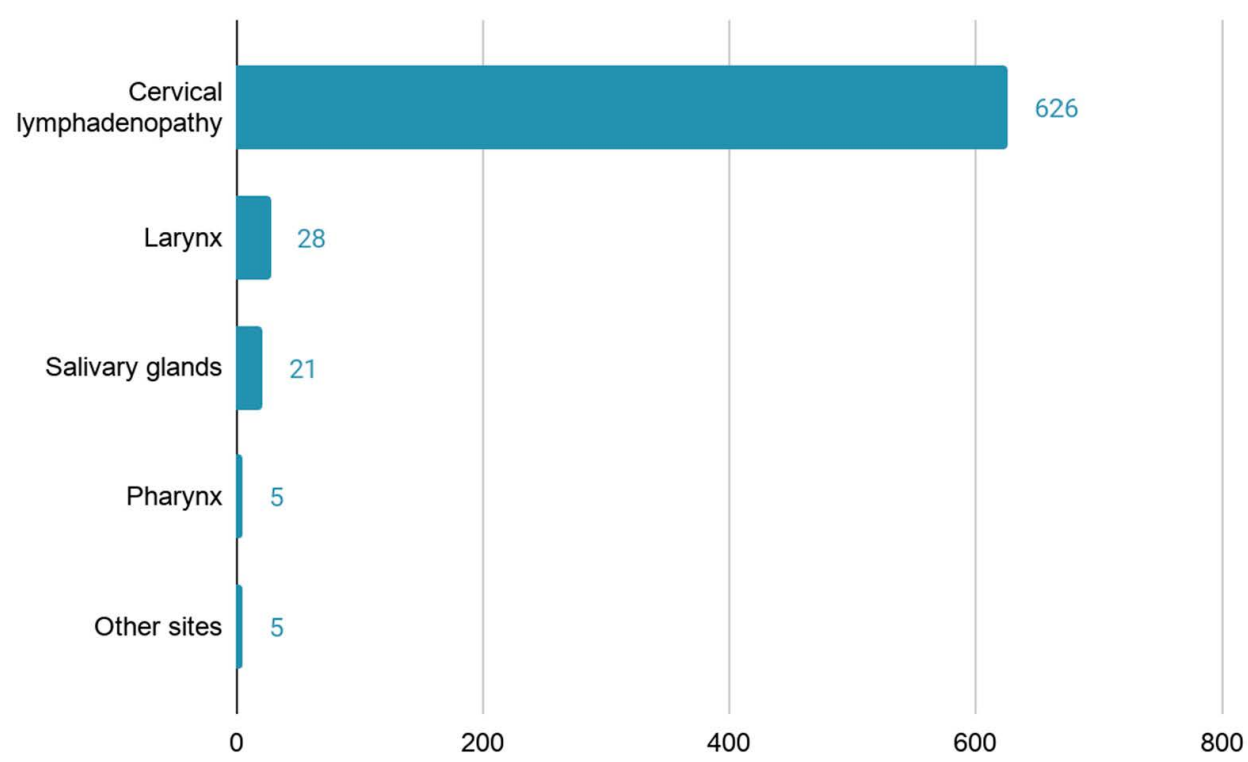

Figure 5. Location of tuberculosis lesions. 


\section{Discussion}

From a clinical and evolutive point of view, syphilis infection is classified into three stages: primary, secondary, and tertiary. The lesions present on the skin and oral mucosa are characteristic of each stage of the disease [15]. Oral lesions are most commonly present on the lips, but other locations include the oral mucosa: tongue, palate, gingiva, and tonsils. The upper lip is more common in men, while lower lip infection is prevalent in women [I5]. The inoculation canker (primary stage of syphilis) is chronic ulceration with a diameter of $\mathrm{I}-\mathrm{I} .5 \mathrm{~cm}$, prominent, with a "clean", reddish-red appearance, and an extremely contagious serous exudate $[\mathrm{I5}, \mathrm{I} 6]$. Classically, the lesions on the lips are the most common extragenital location, present in $60 \%$ of cases $[\mathrm{I} 6]$. However, in the present study, the most common location of oral syphilis was the tongue, followed closely by the lip location. The canker of the labial commissure is ulcerative, painful, and covered with crusts, mimicking hyperplastic angular cheilitis. The most frequent syphilis lesions were encountered in the second stage of the disease in all the analyzed studies. The tongue location-encountered in all stages of syphilis lesions-can show painful, nonspecific ulceration. On the gingival mucosa, the lesions may appear as an ulcer or in the form of a hyperplastic area similar to an epulis. Located on the tonsils, syphilis can simulate a squamous cell carcinoma, also considering the satellite adenopathy which accompanies it [Is]. The clinical appearance of the lesions may be influenced by other additional oral infections, association with HIV infection, or medical history of previous inappropriate treatments. Due to their great polymorphism, luetic lesions can be confused with multiple skin diseases or mucosal disorders in the oral cavity [I5]. Syphilis diagnosis can be confirmed by highlighting spirochetes in the lesion exudate or by darkfield microscopy of a fraction of an active lesion or indirectly by highlighting specific antibodies [2,15].

The signs and symptoms of tuberculosis are often clinically and remarkably blurred. The patient may suffer from episodic fever and chills, but asthenia and malaise are often the main features of the disease. There may also be a gradual weight loss accompanied by a persistent cough with or without associated hemoptysis. Locally, patients report stinging, burning, odynophagia, halitosis, hypersalivation, and bleeding. Trismus and hypoesthesia may accompany osteitis and tuberculous osteomyelitis [7,I5]. In order to diagnose chronic suspected tuberculosis lesions, a biopsy examination is mandatory and it has a reliability of over $90 \%[\mathrm{I} 5]$. The diagnosis of active infection needs confirmation by the organisms' identification on stained smears or the culture of infected tissues or sputum [7]. The definitive diagnosis of tuberculosis is considered the detection of acid-resistant bacilli in the smear of the sputum. Imaging investigations such as pulmonary radiography and tuberculin testing are useful in supplementing the diagnosis of tuberculosis. Tuberculosis of the head and neck area represents a low percentage of $0 . I-5 \%$ of all tuberculosis cases. In order of frequency, the oral areas of most interest are the tongue, jaw, gums, lips, buccal mucosa, soft palate, and hard palate [Is]. The frequency of lesions is $58 \%$ secondary lesions, while primary lesions amount to $42 \%$ [ $\mathrm{I} 5]$. Primary lesions are present at a lower rate because the oral mucosa is rarely a gate for the microorganism. Secondary lesions are caused by dissemination from active or hematogenous lung lesions in the presence of favoring factors: lack of hygiene, smoking, chronic irritants, and altered general state [ $\mathrm{I5}]$. The most common sites are the lips and the buccal mucosa, followed by gingiva. Often, oral ulcerative lesions from tuberculosis coexist with palpable lymph nodes mimicking the presence of squamous cell carcinoma [2]. In the present analysis, cervical lymphadenopathy was the most frequently encountered in tuberculosis in the head and neck region. A great possibility of contracting the infection is in the case of the dentist as it has been shown that acid-resistant microorganisms can be contracted by swabbing or washing the oral cavity of tuberculosis patients [7]. 


\section{Conclusions}

Rare infections of the oral mucosa may occur in daily dental practice. Their diagnosis may be challenging due to their evolving polymorphic clinical appearance and their ability to mimic various diseases in the oral cavity and head and neck area.

Author Contributions: Conceptualization and methodology of the study, I.P. and S.T.; documentation, R.C., I.P.; primary draft Preparation, R.C.; writing and review, I.P., S.T.; visualization and administration, I.P.

Funding: This research received no external funding.

Conflicts of Interest: None declared.

\section{References}

I. Placa, M.L.; Ghersetich, I. Infections of the Oral Cavity. In Oral Diseases; Lotti, T.M., Parish, L.C., Rogers, R.S., Eds.; Springer: Berlin/Heidelberg, Germany, I999; pp. 77-87.

2. Neville, B.W.; Damm, D.D.; Allen, C.M.; Chi, A.C. Oral and Maxillofacial Pathology; Elsevier Health Sciences: St. Louis, MO, USA, 2oI5.

3. Nakashima, A.K.; Rolfs, R.T.; Flock, M.L.; Kilmarx, P.; Greenspan, J.R. Epidemiology of syphilis in the United States, 194I-1993. Sex. Transm. Dis. 1996, 23, 16-23. [CrossRef] [PubMed]

4. Syphilis CDC Facts Sheet. Available online: http://www.cdc.gov/std/statsi4/syphilis.htm (accessed on Io October 20I5).

5. World Health Organization. Global Incidence and Prevalence of Selected Curable Sexually Transmitted Infections-2008; Department of Reproductive Health and Research, World Health Organization: Geneva, Switzerland, 2012.

6. Barrett, A.W.; Villarroel Dorrego, M.; Hodgson, T.A.; Porter, S.R.; Hopper, C.; Argiriadou, A.S.; Speight, P.M. The histopathology of syphilis of the oral mucosa. J. Oral Pathol. Med. 2004, 33, 286-29I. [CrossRef] [PubMed]

7. Sivapathasundharam, B.; Gururaj, N. Bacterial infections of oral cavity. In Shafer's Textbook of Oral Pathology; Elsevier: New Delhi, India, 20I2; pp. 309-326.

8. Centers for Disease Control and Prevention (CDC). Reported Tuberculosis in the United States, 2003; US, Department of Health and Human Services, CDC: Atlanta, GA, USA, 2005.

9. Schuch, L.F.; da Silva, K.D.; de Arruda, J.A.A.; Etges, A.; Gomes, A.P.N.; Mesquita, R.A.; Tarquinio, S.B.C. Forty cases of acquired oral syphilis and a review of the literature. Int. J. Oral Max. Surg. 2019, 48, 635-643. [CrossRef] [PubMed]

Io. Leuci, S.; Martina, S.; Adamo, D.; Ruoppo, E.; Santarelli, A.; Sorrentino, R.; Mignogna, M.D. Oral syphilis: A retrospective analysis of $\mathrm{I2}$ cases and a review of the literature. Oral Dis. 2013, 19, 738-746. [CrossRef] [PubMed]

II. Matias, M.D.P.; Jesus, A.O.D.; Resende, R.G.; Caldeira, P.C.; De Aguiar, M.C.F. Diagnosing acquired syphilis through oral lesions: The I2 year experience of an Oral Medicine Center. Braz. J. Otorbinolaryngol. 2020, 86, 358-363. [CrossRef] [PubMed]

I2. Estomba, C.M.C.; Reinoso, F.A.B.; Schmitz, T.R.; Echeverri, C.C.O.; Cortés, M.J.G.; Hidalgo, C.S. Head and neck tuberculosis: 6-year retrospective study. Acta Oto-Laryngol. 2016, 67, 9-14. [CrossRef]

I3. Qian, X.; Nguyen, D.T.; Albers, A.E.; Dong, Y.; Lyu, J.-X.; Meng, Q.H.; Bi, X.; Graviss, E.A. An eight-year epidemiologic study of head and neck tuberculosis in Texas, USA. Tuberculosis 2019, 116, $\mathrm{S}_{71}-\mathrm{S}_{77}$. [CrossRef] [PubMed]

I4. Pang, P.; Duan, W.; Liu, S.; Bai, S.; Ma, Y.; Li, R.; Sun, C. Clinical study of tuberculosis in the head and neck region-II years' experience and a review of the literature. Emerg. Microbes Infect. 2018, 7, I-IO. [CrossRef] [PubMed]

I5. Țovaru, Ş.; Ţ̧ovaru, M.; Costache, M.; Demarosi, F.; Mihai, L. Medicină şi Patologie Oralăa Qmed. Publishing: Bucureşti, Romania, 2008; Volume II.

16. Bandara, H.M.H.N.; Samaranayake, L.P. Viral, bacterial, and fungal infections of the oral mucosa: Types, incidence, predisposing factors, diagnostic algorithms, and management. Periodontol. 2000 2019, 80, I48-176. [CrossRef] [PubMed] 